\title{
WS15-P03
}

\section{Use of Seismic Interferometry to Improve the Imaging of a Heterogeneous Landfill}

\author{
L.A. Konstantaki (Delft University of Technology), D. Draganov* (Delft \\ University of Technology), R. Ghose (Delft University of Technology) \& T. \\ Heimovaara (Delft University of Technology)
}

\section{SUMMARY}

In this study we investigate the application of seismic interferometry (SI) to seismic reflection data recorded over a landfill. Landfills represent strongly heterogeneous subsurfaces making the seismic reflection imaging challenging. We show that SI improves the imaging of high-density areas, which create scattering events in the recorded data. In addition, we use SI to subsequently predict surface-wave energy and remove it from the original data to improve the imaging of reflectors. The combined interpretation of the conventional reflection seismic data, SI and reflection data with surface waves removed improves the understanding of the landfill body. 


\section{Introduction}

Seismic reflection imaging of a heterogeneous subsurface, such as a landfill, is challenging. We propose the use of seismic interferometry (SI) to improve the imaging of a landfill body. Previous seismic studies of landfills have shown that diffractions and background noise mask the reflection signal (e.g., De Iaco et al. 2003). Using numerically modelled data, Konstantaki et al. (2013) showed that SI improves the imaging of shallow scatterers in a landfill. We now apply SI to conventional reflection seismic survey (CRSS) data obtained on a landfill and investigate the advantages of SI. Konstantaki et al. (2015) propose a processing procedure for the CRSS data from a landfill and remark on the difficulty of interpreting near-surface reflections due to the dominant presence of surface waves. Here, we also investigate the removal of surface waves using SI for landfill application.

High-density areas in the landfill body are the reason for the presence of strong scattering events in the seismic data. Such areas are of specific interest to the landfill operators, because they can act as obstructions to the leachate flow and thus affect the treatment of the landfill. Reliable imaging and characterization of the landfill body is important for the improvement of treatment technologies (Powrie and Beaven 1999). Characterization of the subsurface is necessary for the calculation of future emissions from the landfill (McDougall and Fleming 2013). Consequently, it is important to improve the imaging of the CRSS data.

\section{Data acquisition and processing}

In the summer of 2013, we performed field experiments in the Wieringermeer landfill, the Netherlands. We acquired seismic reflection shear (S) wave data using an S-wave high-frequency vibrator (Ghose et al. 1996). The 48 horizontal geophones that we planted had a $0.5 \mathrm{~m}$ spacing and they were kept fixed. The shot spacing was $1 \mathrm{~m}$. Further details regarding the acquisition parameters can be found in Konstantaki et al. (2015). Figure 1 shows the data acquisition geometry.
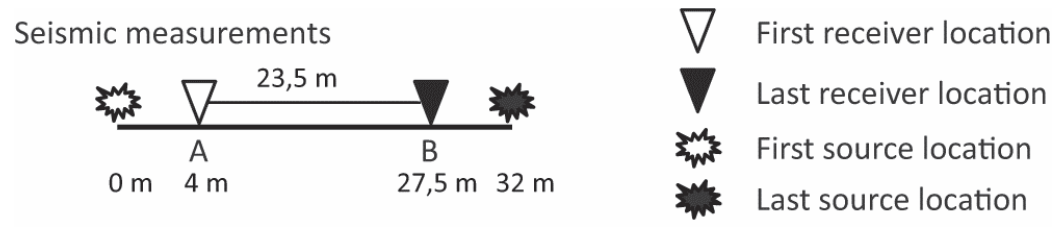

Figure 1 Data-acquisition geometry (adapted from Konstantaki et al. (2015)).

We apply SI to the CRSS data that we recorded. For this, we do the following. (a) In order to enhance the later arrivals we multiply the CRSS data by $\mathrm{e}^{1.3^{*} \mathrm{t}}(\mathrm{t}$ is time). (b) The direct arrivals do not contain any reflection information; therefore, we apply top mute to remove them. (c) We sort the data into common-received gathers (CRG). (d) Apply cross-correlation and summation over all sources. (e) For removal of low- and high-frequency noise, we apply a bandpass filter (5-35-95-110 Hz), we also apply a notch filter to remove $50-\mathrm{Hz}$ power line noise. (f) Depending on the geometry and the subsurface, reflections could be retrieved at positive, negative, or at both times. To obtain a final virtual-shot gather we choose positive, negative, or a summation of positive and negative times according to the specific source-received positions. (g) Finally, we process the SI data in a way similar to the CRSS data (Konstantaki et al. 2015). All stacked sections have a post-stack bandpass filter of 10-35-95-110 Hz applied.

Surface waves were a challenge in the processing of the CRSS data (Konstantaki et al. 2015). Here, we attempt to remove the surface-wave energy using SI. The goal is to reconstruct the surface waves using SI and then adaptively subtract them from the original CRSS data. For the preparation of the data with retrieved surface waves $\left(\mathrm{SI}^{\mathrm{S}}\right)$, we perform step (a) as described above, followed by a bandpass frequency filter $(5-35-95-110 \mathrm{~Hz})$ to enhance the surface waves. Next, we apply steps (b) to (d) and step (f). Finally, in order to obtain shot gathers after adaptive subtraction (AS), we subtract, 
using a least-squares matching filter (Verschuur et al. 1992), the $\mathrm{SI}^{\mathrm{S}}$ shot gathers from the corresponding CRSS shot gathers. The matching filter is 21 sample points long inside a 100 sample points time window and extends spatially over 5 traces. To retrieve the AS stacked section, we process the data in a way similar to the CRSS data.

\section{Results}

Figure 2 shows the shot gathers at horizontal location $22 \mathrm{~m}$ for the CRSS, SI, and AS data. Figures $2 a, c, e$ depict the raw data, whereas Figure 2b,d,f the processed data for the three cases. The greyshaded areas show interpreted reflections, the red and green hyperbolas are interpreted scattering events: red are scattering events interpreted in the CRSS shot gather (Konstantaki et al. 2015), whereas green are interpreted in the SI shot gather. Comparing Figure $2 b$ and $2 d$ we observe that scattering events 1,2,5,6 are present in both results. However, scattering events 5 and 6 would not be interpreted in the CRSS shot gather without the information from the SI shot gather. Scattering events 3 and 4 represent the same event, but appear with reversed polarity. In the SI shot gather, the reflections are more continuous and coherent. Comparing the gathers in Figures $2 \mathrm{~b}, \mathrm{~d}, \mathrm{f}$ we see further improvement of the reflections in Figure 2f: the adaptive subtraction has succeeded in removing surface-wave energy. However, the $\mathrm{S} / \mathrm{N}$ ratio has reduced after AS, meaning that the filter does not remove all surface-wave energy. The scattering events in the AS result appear worse and have been affected by the predictive subtraction.

The stacked sections produced from these three strategies of processing (Figure 3) strengthen the above observations. SI exhibits a higher degree of scatterers (Figure 3d). Having a higher number of traces, SI is able to: (a) image shallow scatterers better (Konstantaki et al. 2013), e.g., scatterer at location $9.25 \mathrm{~m}$ and time $25 \mathrm{~ms}$ is now identifiable; (b) image scatterers on reflectors (e.g., 5 and 6) scatterers on reflectors are less interpreted in the CRSS data, as noise and insufficient stacking power do not allow distinction between reflectors and scatterers in a very heterogeneous subsurface; (c) define the correct apex of the scatterer (e.g., opposite-polarity effect - yellow rectangles). Diffractions in both SI and CRSS stacked images will not be flattened during the stacking, because we use rootmean-square velocities. However, the extra information obtained in SI allows for better imaging. Comparing the CRSS and AS stacked sections (Figure $3 \mathrm{~b}$ and $3 \mathrm{f}$ ), we observe the improvement of the reflectors in the AS section. The grey-shaded areas show the continuous reflectors interpreted on the AS section. The yellow-shaded line shows the bottom of the landfill, which can now be readily interpreted. Most of the red scatterers are interpretable in the AS section, though the green ones are less well imaged.

\section{Conclusions}

We tested the application of seismic interferometry (SI) on field seismic reflection data obtained above a heterogeneous landfill. We showed that SI improves the imaging of the higher-density areas (scatterers) and that adaptive subtraction (AS) of surface waves predicted by SI results in improved imaging of the reflectors.

SI might result in retrieved artifacts. AS is of lower $\mathrm{S} / \mathrm{N}$ ratio than the original conventional reflection seismic survey (CRSS) data; however, a combined interpretation of all three results (CRSS, SI, and AS) allows for a better understanding of the landfill heterogeneity. In terms of characterization of the landfill, the obtained velocity models from data processing of the CRSS, SI and AS data improve the final velocity model and thus the characterization of the landfill.

\section{Acknowledgments}

This research is supported by the Dutch Technology Foundation (STW) under the project number 11035. The research of Deyan Draganov is supported by the Division for Earth and Life Sciences $(A L W)$ with financial aid from the Netherlands Organization for Scientific Research (NWO). Many 
thanks to Alber Hemstede, Michael Afanasyev, Shirish Baviskar, Andre van Turnhout, Asiya Kudarova and Andiry Bun for their help in the acquisition of field data.

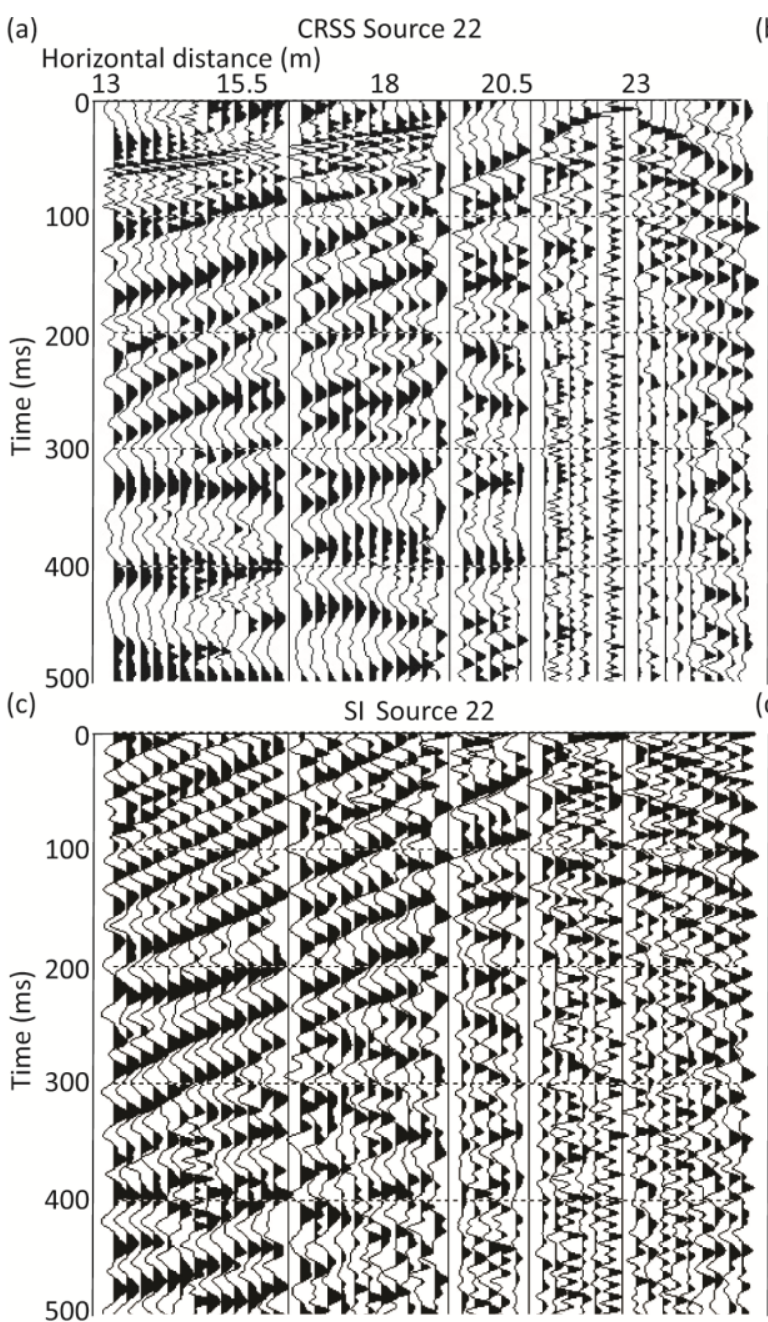

(b) CRSS Source 22

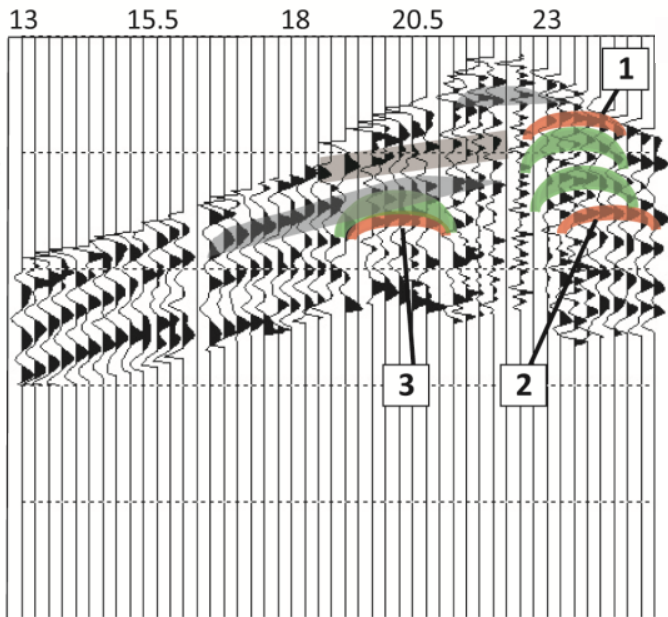

(d)

SI Source 22

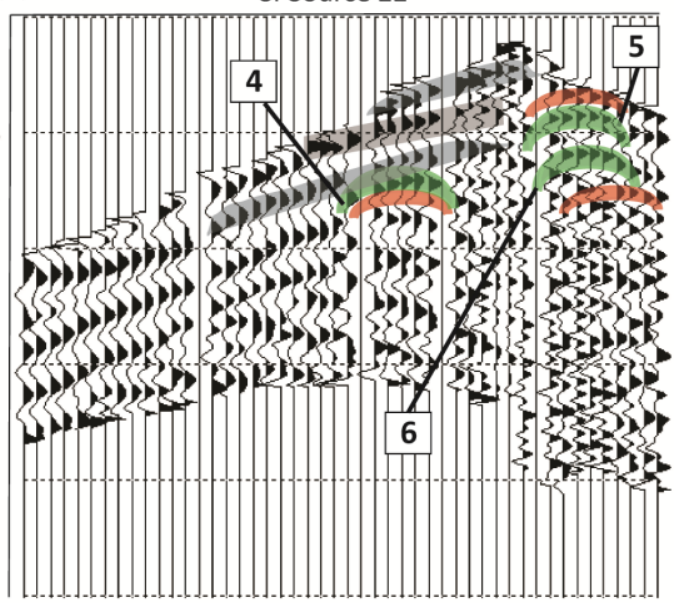

(e)

AS Source 22

AS Source 22
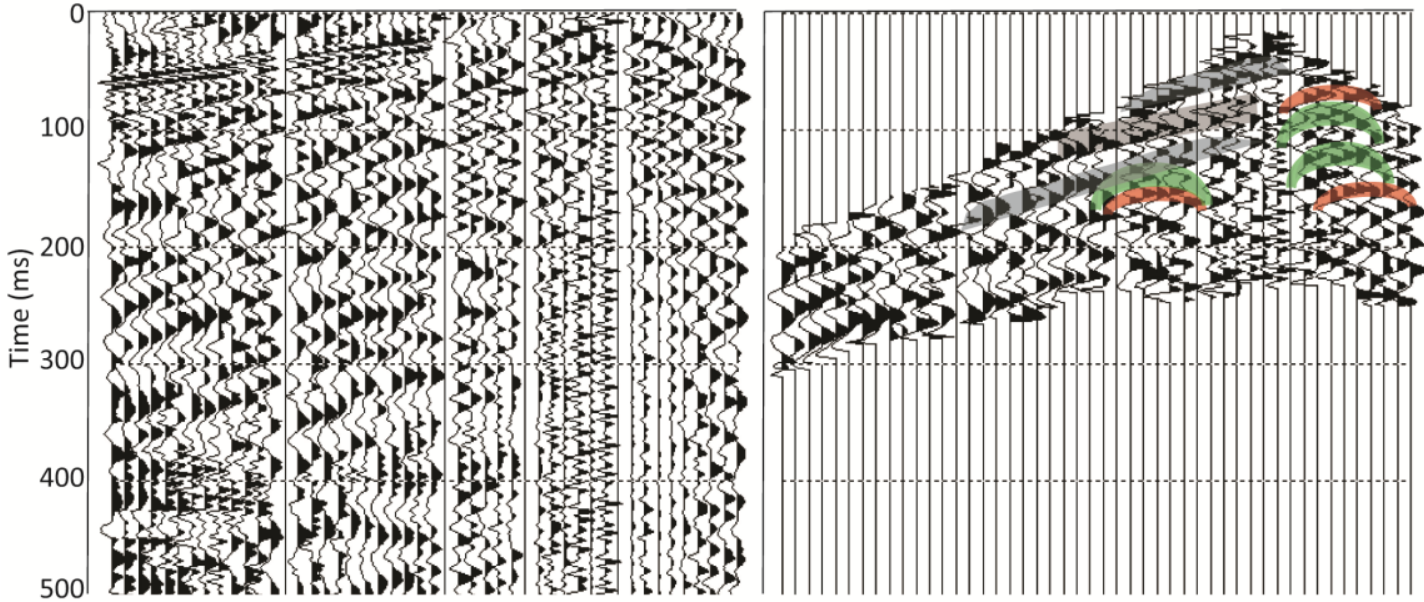

Figure 2 Shot gather for a source at horizontal location $22 \mathrm{~m}$ obtained from (a) conventional reflection seismic survey (CRSS), (c) seismic interferometry (SI), (e) adaptive subtraction (AS). Processed data and interpretation for (b) CRSS, (d) SI, and (f) AS. The grey-shaded areas show reflections, whereas the green and red hyperbolas show scattering events. 


\section{C.. MADRID 2015}

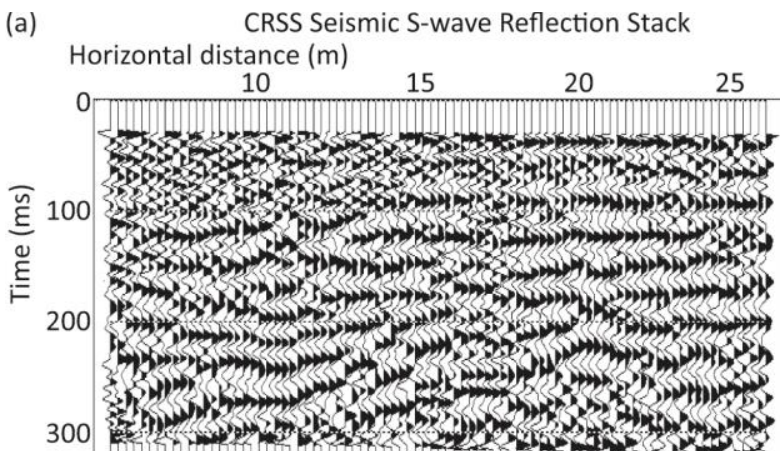

(b) CRSS Seismic S-wave Reflection Stack
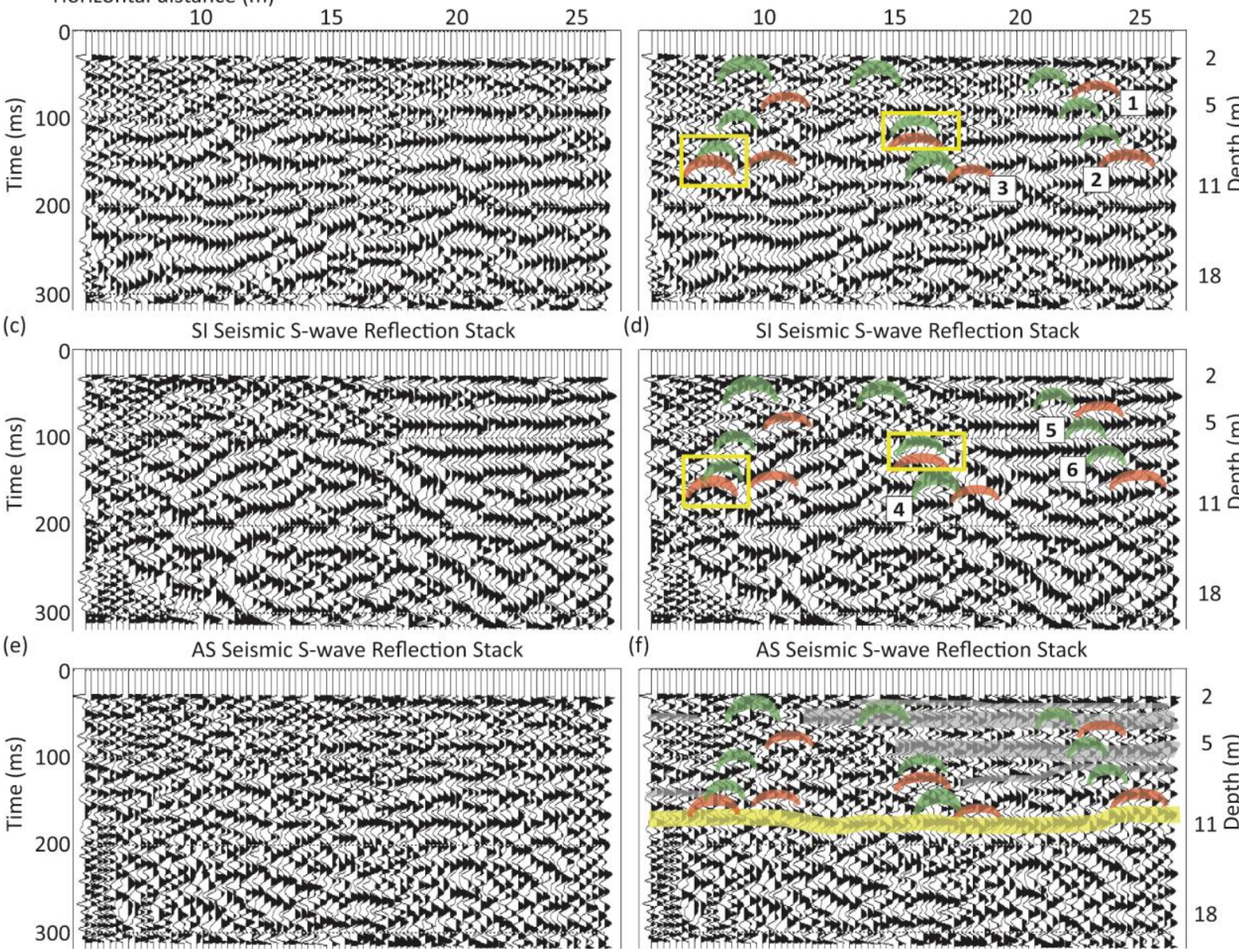

(d)

SI Seismic S-wave Reflection Stack

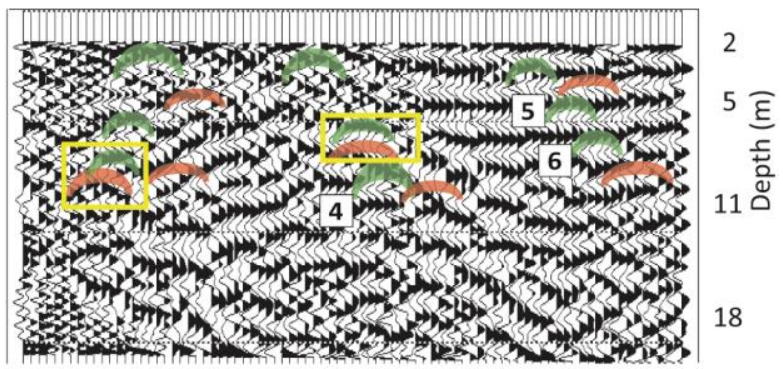

(f)

AS Seismic S-wave Reflection Stack

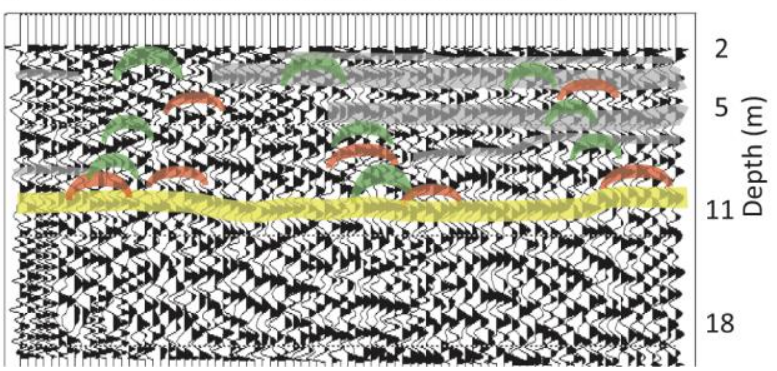

Figure 3 Stacked sections obtained from (a) conventional reflection seismic survey (CRSS) data, (c) seismic interferometry (SI) data, (e) adaptive subtraction data (AS). Interpretation for (b) CRSS, (d) SI, and (f) AS. Red and green hyperbolas show scatterer locations, grey-shaded areas show reflectors, and the yellow line shows the bottom of the landfill.

\section{References}

De Iaco, R., Green, A. G., Maurer, H. R. and Horstmeyer, H. [2003] A combined seismic reflection and refraction study of a landfill and its host sediments. Journal of Applied Geophysics, 52(4), 139-156.

Ghose, R., Brouwer, J. and Nijhof, V. [1996] A portable S-wave vibrator for high-resolution imaging of the shallow subsurface. 58th Annual International Conference and 20 Exposition, EAGE, Extended Abstracts, M037.

Konstantaki, L.A., Draganov, D., Heimovaara, T. and Ghose, R. [2013] Imaging scatterers in landfills using seismic interferometry. Geophysics, 78(6), 1-10. doi:10.1190/GEO2013-0099.1

Konstantaki, L.A., Ghose, R., Draganov, D., Diaferia, G. and Heimovaara, T. [2015] Characterization of a heterogeneous landfill using seismic and electrical resistivity data. Geophysics, 80(1), 113, doi:10.1190/GEO2014-0263.1

McDougall, J. R. and Fleming, I. R. [2013] Hydro-bio-mechanical modelling of filling and post closure landfill behaviour - a case study. HPM5 - Fifth International workshop on Hydrophysico-mechanics of landfills, 77-87.

Powrie, W. and Beaven, R. P. [1999] Hydraulic properties of household waste and implications for landfills. Proceedings of the ICE - Geotechnical Engineering, 137(4), 235-247.

Verschuur, D.J., Berkhout, A.J. and Wapenaar, C.P.A. [1992] Adaptive surface-related multiple elimination. Geophysics, 57(9), 1166-1177. doi:10.1190/1.1443330 